\title{
A search for millimeter emission from the coldest and closest brown dwarf with ALMA
}

\author{
Dirk Petry and Valentin D. Ivanov \\ European Southern Observatory, Karl-Schwarzschild-Str. 2, 85748 Garching, Germany \\ e-mail: dpetry@eso.org
}

Received 5 June 2020 / Accepted 5 August 2020

\begin{abstract}
Context. WISE J085510.83-071442.5 (W0855) is a unique object: with $T_{\mathrm{eff}} \approx 250 \mathrm{~K}$ it is the coldest known brown dwarf (BD), located at only $\approx 2.2 \mathrm{pc}$ form the Sun. It is extremely faint, which makes any astronomical observations difficult. However, at least one remotely similar ultra-low-mass object, the M9 dwarf TVLM 513-46546, has been shown to be a steady radio emitter at frequencies up to $95 \mathrm{GHz}$ with superimposed active states where strong, pulsed emission is observed.

Aims. Our goal is to determine the millimeter radio properties of W0855 with deep observations around $93 \mathrm{GHz}(3.2 \mathrm{~mm})$ in order to investigate whether radio astrometry of this object is feasible and to measure or set an upper limit on its magnetic field.

Methods. We observed W0855 for $94 \mathrm{~min}$ at $85.1-100.9 \mathrm{GHz}$ on 24 December 2019 using 44 of the Atacama Large millimeter Array (ALMA) $12 \mathrm{~m}$ antennas. We used the standard ALMA calibration procedure and created the final image for our analysis by accommodating the Quasar 3C 209, the brightest nearby object by far. Furthermore, we created a light curve with a $30 \mathrm{~s}$ time resolution to search for pulsed emission.

Results. Our observations achieve a noise RMS of $7.3 \mu \mathrm{Jy}_{\text {beam }}{ }^{-1}$ for steady emission and of $88 \mu \mathrm{Jy}$ for $30 \mathrm{~s}$ pulses in the aggregated bandwidth (Stokes $I$ ). There is no evidence for steady or pulsed emission from the object at the time of the observation. We derive $3 \sigma$ upper limits of $21.9 \mu \mathrm{Jy}$ on the steady emission and of $264 \mu \mathrm{Jy}$ on the pulsed emission of W0855 between $85 \mathrm{GHz}$ and $101 \mathrm{GHz}$.

Conclusions. Together with the recent non-detection of W0855 at 4-8 GHz, our constraints on the steady and pulsed emission from W0855 confirm that the object is neither radio-loud nor magnetospherically particularly active.
\end{abstract}

Key words. stars: evolution - brown dwarfs - stars: individual: WISE J085510.83-071442.5 - stars: magnetic field

\section{Introduction}

Luhman $(2013,2014)$ discovered three new brown dwarfs (BDs) in two systems (one is a binary) in the immediate vicinity of the Sun, and there are reports for other BDs that are not much more distant (e.g. Scholz 2014; Faherty et al. 2018; Tinney et al. 2018; Kirkpatrick et al. 2019; Marocco et al. 2019; Meisner et al. 2020). This indicates that these objects may be very common in the Milky Way. As Wright et al. (2014) suggested, the all-sky survey carried out by the Wide-field Infrared Survey Explorer (WISE; Wright et al. 2010) may contain more of them.

The low temperatures of BDs make them intrinsically extremely faint, even in the mid-infrared (IR), where their spectral energy distributions (SEDs) peak. WISE J085510.83071442.5 (W0855 hereafter) with $T_{\text {eff }} \approx 250 \mathrm{~K}$ (Luhman 2014; Beamín et al. 2014; Kirkpatrick et al. 2019) is the coldest known BD. Its temperature places it at the intersection between giant planets in the Solar System (Jupiter is not very different with $T \sim 140-170 \mathrm{~K}$ ), cool exoplanets at large orbital distances in other planetary systems, and the ultracool BDs and free-floating planets at $T \sim 400 \mathrm{~K}$ or higher (Beichman et al. 2013).

Located as close as $\approx 2.2 \mathrm{pc}$ to us, W0855 presents a unique opportunity to study an ultracool object and its environment in detail. Considerable effort was made to observe it from the ground (Kopytova et al. 2014; Beamín et al. 2014), but it yielded only one marginal detection (Faherty et al. 2014) and the construction of an SED had to wait until some space-based observations were collected (Luhman 2013, 2014; Luhman \& Esplin 2016; Zapatero Osorio et al. 2016). They confirmed the initial temperature estimate of $250 \mathrm{~K}$.
Skemer et al. (2016) and Morley et al. (2018) obtained spectra of W0855 at $\lambda \approx 3-4 \mu \mathrm{m}$. A comparison with Jupiter suggests a lack of $\mathrm{PH}_{3}$ absorption (Kunde et al. 1982). This molecule is unstable; phosphorus is easily captured in $\mathrm{P}_{4} \mathrm{O}_{6}$. The existence of $\mathrm{PH}_{3}$ in the Jupiter atmosphere is evidence for vertical mixing between the hot interior and cooler outer parts of the planet. The lack of $\mathrm{PH}_{3}$ in W0855 may indicate that it has a more quiescent atmosphere than Jupiter. The reported modelling of these spectra suggests that $\mathrm{CH}_{4}$ is present, but at sub-solar abundance.

Low-mass stars and BDs offer a shortcut towards finding small planets, comparable to the Earth, because of their advantageous planet-to-host mass and luminosity ratios. Not surprisingly, the first directly imaged exoplanet orbits a BD (Chauvin et al. 2004, 2005) and the nearest planet was discovered with radial velocity monitoring of the M-type star (Anglada-Escudé et al. 2016). Hubble Space Telescope (HST) imaging found no companions of W0855 within 0.5 AU (Luhman \& Esplin 2016; Zapatero Osorio et al. 2016) and Spitzer imaging reported none within 9-970 AU (Melso et al. 2015). Unfortunately, the object is too faint for radial velocity monitoring even with the best current or near-future spectrographs. This makes astrometry the most promising tool to search for companions, likely of planetary mass, around W0855. Because these companions do not need to be detected directly, the astrometric method can probe smaller separations than direct imaging. Luhman \& Esplin (2016) monitored W0855 with Spitzer (see also Esplin \& Luhman 2016). They measured accurate parallax and proper motion, but found no indications for companions.

Another possibility is radio astrometry: Stars and planets are well known to emit in the radio. Typically, radio 
astrometry has been applied to evolved or cool and active objects (e.g. Gawroński et al. 2018; Curiel et al. 2019). Berger et al. (2001) opened the field of BD radio observations by reporting a radio detection of LP 944-20 (see also Sheehan et al. 2019). Radio interferometers deliver higher angular resolution and higher positional accuracy than the optical and IR instruments (Xu et al. 2019). These advantages were demonstrated in radio-astrometric campaigns on $\mathbf{M}$ dwarfs by Bower et al. (2009), and on BDs by Forbrich \& Berger (2009), Forbrich et al. (2013), and Gawroński et al. (2017).

However, radio astrometry is not always feasible. Depending on the level of activity, the radio emission can vary widely. This prompted us in 2018 to investigate whether W0855 might be accessible by the most sensitive millimeter $(\mathrm{mm})$ observatory available today, ALMA ${ }^{1}$, within a reasonable amount of observing time.

The exact mechanisms of radio emission in ultracool BD are unclear, but the best available models (e.g. Nichols et al. 2012) involve strong magnetic fields and plasma outflows. A detection would indicate that these can be present in the atmospheres of objects in the $T_{\text {eff }} \sim 250 \mathrm{~K}$ regime.

Assuming W0855 is a fast rotator with a strong magnetic field, we can estimate its radio properties by scaling the apparent flux of potentially similar objects. The only at least remotely similar object with a detection at $\mathrm{mm}$ wavelengths is the M9 dwarf TVLM 513-46546 (distance $D=10.8$ pc, TVLM 513 hereafter), that has been detected with ALMA at the $56 \pm 12 \mu \mathrm{Jy}$ level by Williams et al. (2015). It would yield an apparent flux of $\approx 1.3 \mathrm{mJy}$ at $93 \mathrm{GHz}$ (Band 3) at the distance of W0855. It is considerably hotter than W0855, but the origin of the mm emission is not thermal, as discussed below. The other example mentioned above, the BD LP944-20 ( $D=6.4 \mathrm{pc}$; Berger et al. 2001), with an apparent flux density of $\approx 80 \mu \mathrm{Jy}$ at $8.5 \mathrm{GHz}$, would yield a flux density of $\approx 0.7 \mathrm{mJy}$ at the distance of W0855. These are both optimistic estimates because only a fraction of BDs show such a high level of activity. Those that do are mostly young, as suggested by the presence of Li in LP944-20 and its dusty clouds (Pavlenko et al. 2007), but the age of W0855 is still unconstrained. Fluxes of the order of $1 \mathrm{mJy}$ are easily within reach: even tentatively adopting a lower apparent flux by about a factor of 50 (equivalent to a more modest activity level) yields a $3 \sigma$ detection (i.e. $1 \sigma$ sensitivity of $0.0087 \mathrm{mJy}$ ) in $\approx 2 \mathrm{~h}$ integration in ALMA Band 3.

A radio-quiet W0855 is beyond reach: an unresolved (the apparent angular diameter of a Jupiter-sized BD at the distance of W0855 is 0.21 mas) black body of $250 \mathrm{~K}$ at $2.2 \mathrm{pc}$ has a flux density of $0.26 \mu \mathrm{Jy}$ at $100 \mathrm{GHz}$, requiring $\mathrm{a} \approx 2 \mathrm{yr}$ integration to achieve a $3 \sigma$ detection.

After the second iteration of our proposal to observe W0855 with ALMA had already been approved, however, Kao et al. (2019) reported for W0855 a VLA 4-8 GHz upper limit on the steady flux of $7.2 \mu \mathrm{Jy}$. With this new information, it was unlikely that we would detect steady emission from W0855 at $9 \mathrm{GHz}$ if its spectrum is similar to that of TVLM 513, which shows a slow decay between $8 \mathrm{GHz}$ and ALMA Band 3. However, both TVLM 513 and other ultracool dwarfs have also been reported to have states of high activity where they produce bright broadband pulses of polarised radio emission (e.g. Hallinan et al. 2007, 2008) with pulse durations of the order of minutes to hours and substructures down to the $30 \mathrm{~s}$ level. These are thought to be related to the electron cyclotron maser instability (ECMI) in the magnetosphere and thus coupled to the rota-

\footnotetext{
1 http://almascience.org
}

tional periods of the objects. The pulses are visible both in full intensity (Stokes $I$ ) and circularly polarised emission (Stokes $V$ ) and reach fluxes of $4 \mathrm{mJy}$ at $8.4 \mathrm{GHz}$. Scaled to the (shorter) distance of W0855 and extrapolated to $93 \mathrm{GHz}$ using the measured spectrum of TVLM 513, such pulses could reach more than $10 \mathrm{mJy}$ for W0855. With the sensitivity of ca. $0.1 \mathrm{mJy}$ of our ALMA observation for $30 \mathrm{~s}$ integration time, detecting W0855 in such an active state would still be possible. However, a detection of pulses at $93 \mathrm{GHz}$ would imply the presence of exceptionally strong magnetic fields in excess of $34 \mathrm{kG}$ if they were to be explained by the ECMI mechanism.

\section{Observations}

\subsection{Band 3 observations in December 2019}

The observations were carried out between 2019-12-24, 5:01:06 UT and 2019-12-24, 7:15:23 UT with 44 of the ALMA $12 \mathrm{~m}$ antennas. Two ALMA execution blocks with $47 \mathrm{~min}$ onsource time each were carried out, yielding a total on-source time of $94 \mathrm{~min}$. The configuration was nominally C43-2. The longest baseline was $313 \mathrm{~m}$, and the shortest baseline was $15 \mathrm{~m}$ long. This resulted in a synthesised beam of ca. $3^{\prime \prime}$ diameter, which represents our angular resolution (see the next section for the exact value). The precipitable water vapour (PWV) in the atmosphere above ALMA was between $3.7 \mathrm{~mm}$ and $4.2 \mathrm{~mm}$ during the observations (reasonably good values for Band 3 ).

The observations were scheduled as standard low-resolution (Time Division Mode) observations with four spectral windows of 128 channels of $15.625 \mathrm{MHz}$ width each. The first and the last eight channels of each spectral window were discarded for low sensitivity, leaving a total effective bandwidth of $7 \mathrm{GHz}$ in the range from $85.1382 \mathrm{GHz}$ to $100.8618 \mathrm{GHz}$.

We had added 3C 209 (PKS J0855-0715, $\alpha=08: 55: 09.5$, $\delta=-07: 15: 03$, J2000; Douglas et al. 1996) as a check source to the observing schedule because the proper motion of W0855 takes it close to this bright blazar during ALMA Cycle 7. Check sources are briefly visited during observations for phase and flux calibration. We explicitly included the object in the image deconvolution process to improve our sensitivity (see next section).

During the observations of the target, the phase centre of the interferometer was kept at the position of W0855, taking the proper motion as measured by Luhman \& Esplin (2016) into account: $\alpha=08: 55: 05.639915, \delta=-07: 14: 36.19233$ (J2000) at the beginning of the first block execution and $\alpha=08: 55: 05.639827$, $\delta=-07: 14: 36.19227$ (J2000) at the beginning of the second. Over the course of the observation, there was therefore no significant movement of the target compared to our angular resolution. The target elevation was between $56.1^{\circ}$ and $74.5^{\circ}$.

The check source 3C 209 was observed during ten 1 min scans over the course of the execution block. Phase, bandpass, and flux calibrators were observed following the standard ALMA calibration procedure. As bandpass and flux calibrator served QSO J0725-0054, as phase calibrator QSO J0847-0703. The typical systematic uncertainty on the standard ALMA flux calibration is better than $10 \%$ in Band 3 (Remijan et al. 2019).

\subsection{Data analysis}

The calibration of the data followed the standard ALMA Quality Assurance procedure for Cycle 7 (see Petry et al. 2014; Remijan et al. 2019) using the calibration pipeline 42866 (ALMA Pipeline Team 2019) based on the CASA data analysis package version 5.6.1-8 (Emonts et al. 2019). The calibrated data were then imaged with the tclean task of the same CASA 


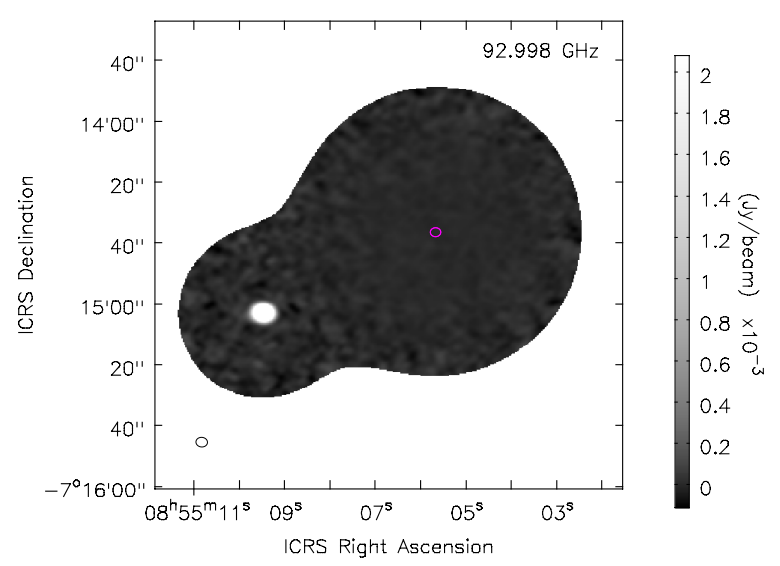

Fig. 1. Map of the W0855 field obtained in this analysis. The expected location of the source is indicated with a magenta ellipse of the shape of the synthesised beam. The bright source to the east is the background quasar 3C 209 with a flux of ca. $20 \mathrm{mJy}$, i.e. a factor of 2740 brighter than the sensitivity of $7.3 \mu \mathrm{Jy}$ that is achieved in the vicinity of W0855. To give an impression of the homogeneity of the noise, the brightness scaling in this high-dynamic-range image was set to strongly overexpose the quasar, which therefore appears more extended than the beam. The beam size is shown again as an ellipse in the lower left corner.

package in mfs mode, that is, combining all spectral channels into one image. At first, only the W0855 data were imaged as a single field with a pixel size of $0.4^{\prime \prime}$ and natural weighting in order to optimise the point-source sensitivity. This resulted in a noise RMS in the central region of the image of $11.8 \mu \mathrm{Jy}$, significantly worse than the expected $8 \mu \mathrm{Jy}$.

We suspected that the loss in sensitivity was due to the sidelobes of the relatively bright nearby object 3C 209 and therefore re-imaged the target by combining the check-source field containing 3C 209 with the target field as a mosaic (but otherwise identical imaging parameters). This permitted us to remove the sidelobes of 3C 209 in the deconvolution and reduced the noise RMS in the region around the nominal position of W0855 to $7.3 \mu \mathrm{Jy}$. The synthesised beam of the observation was measured to be $3.081^{\prime \prime} \times 2.559^{\prime \prime}$. The resulting image is shown in Fig. 1 .

\subsection{Derivation of an upper limit on the steady flux}

To derive a $3 \sigma$ upper limit on the steady (quiescent) flux of W0855 between $85.1 \mathrm{GHz}$ and $100.9 \mathrm{GHz}$, we follow the prescription of Kao et al. (2019; who simply multiplied the measured noise RMS by a factor 3) because we wish to plot our result together with theirs; see Masci (2011) for an alternative, more conservative method. With our noise RMS of $7.3 \mu \mathrm{Jy}$ from above, we obtain an upper limit of $21.9 \mu \mathrm{Jy}$.

\subsection{Search for pulsed emission}

Based on the minimum duration of the pulse structures seen in TVLM 513 (Hallinan et al. 2007), we divided our dataset into $30 \mathrm{~s}$ time bins for which we obtained individual images. Measuring the flux in a beam-shaped region around the W0855 position in each of the 188 images, we derived a light curve (Fig. 2). The distribution of the $30 \mathrm{~s}$ fluxes is consistent with a Gaussian with zero average and RMS $82 \mu \mathrm{Jy}$, which in turn is consistent with the noise RMS of $88 \mu \mathrm{Jy}$ that we measured on average in the individual $30 \mathrm{~s}$ images. A $3 \sigma$ upper limit on the pulsed flux from W0855 between $85.1 \mathrm{GHz}$ and $100.9 \mathrm{GHz}$ during the time of the observation can be placed at $264 \mu \mathrm{Jy}$.

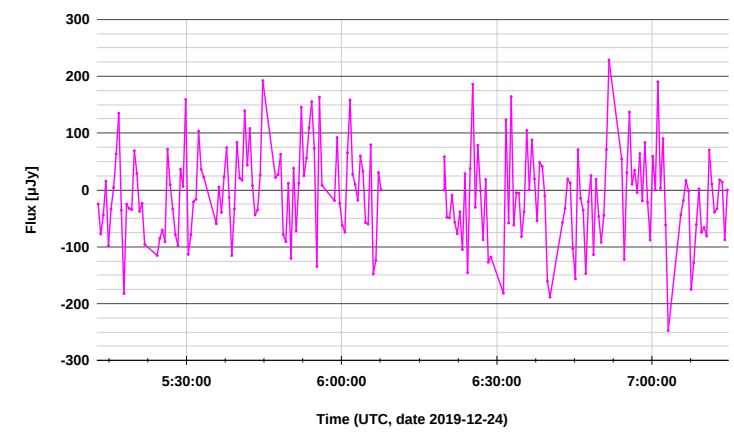

Fig. 2. Light curve of W0855 at $85.1-100.9 \mathrm{GHz}$ (Stokes $I$ ) with $30 \mathrm{~s}$ time resolution obtained in this work. There is no evidence for pulsed emission. The pulse distribution is consistent with a Gaussian and has a mean of $-8.6 \mu \mathrm{Jy}$ and an RMS of $82 \mu \mathrm{Jy}$.

\section{Discussion}

\subsection{Radio emission mechanisms in ultracool objects}

The BD LP944-20 (M9, $D=6.4$ pc; Sheehan et al. 2019) emits at $80 \mu \mathrm{Jy}$ in quiescence and at $3 \mathrm{mJy}$ at peak flare at $8.5 \mathrm{GHz}$ (where the SED of the flaring emission peaks). This is three orders of magnitude too high for stellar coronal emission as described by the empirical relation of Guedel \& Benz (1993), indicating that an additional mechanism that powers the radio emission is at work in ultracool objects. Burningham et al. (2016) provided a recent summary: the activity indicators in the $\mathrm{X}$-ray and optical suggest a rapid activity dropoff at ultracool temperatures, but the radio emission is not reduced as much as the emission in other wavelength ranges. Radio emission has been detected in 5-10\% of the M9-T6 type BDs, which shows a break in the X-ray-to-radio relation in the hotter stellar regime.

The radio detections fall into two categories: (1) pulsed, probably rotationally modulated, $100 \%$ polarised, probably originating in ECMIs (Treumann 2006), and (2) quiescent nonpolarised, always present when pulsed emission is detected; a probable origin is depolarised pulsed emission (Hallinan et al. 2008) or gyrosynchrotron emission (Berger 2002).

Theoretical models by Nichols et al. (2012) suggest that the ECMI in ultracool objects may originate from an upward magnetic field component of the magnetosphere-ionosphere coupling, flowing as a result of a meridional angular velocity gradient in the ionospheric plasma. This is similar to the auroral oval in Jupiter (Grodent et al. 2003) where the flow is driven by the outward diffusion of plasma generated by the motion of Io within the magnetosphere of Jupiter (Hill 1979).

\subsection{The magnetic field of W0855}

A detection of ECMI-generated radio emission would permit us to set a direct lower limit on the magnetic field from the cut-off at the electron cyclotron resonance frequency (Eq. (1) in Williams 2018). A VLA detection at $4-8 \mathrm{GHz}$ implies a magnetic field of at least $B \geq 2 \mathrm{kG}$ (Kao et al. 2016), and only LOFAR and GMRT, which work at $\mathrm{MHz}$ frequencies, have the potential to probe weaker magnetic fields, but such observations of ultracool BDs have proven to be challenging (Burningham et al. 2016; Zic et al. 2019). An ALMA detection at $85-101 \mathrm{GHz}$ would place a lower limit at $B \geq 34 \mathrm{kG}$, an essentially unphysical value for lowmass stars (Reiners \& Basri 2007). A non-detection of ECMI-like pulses therefore supports the ECMI origin of such pulses seen at lower frequencies in other objects. For the BD TVLM 513, a surface magnetic field strength of $B \sim 3 \mathrm{kG}$ was measured based 


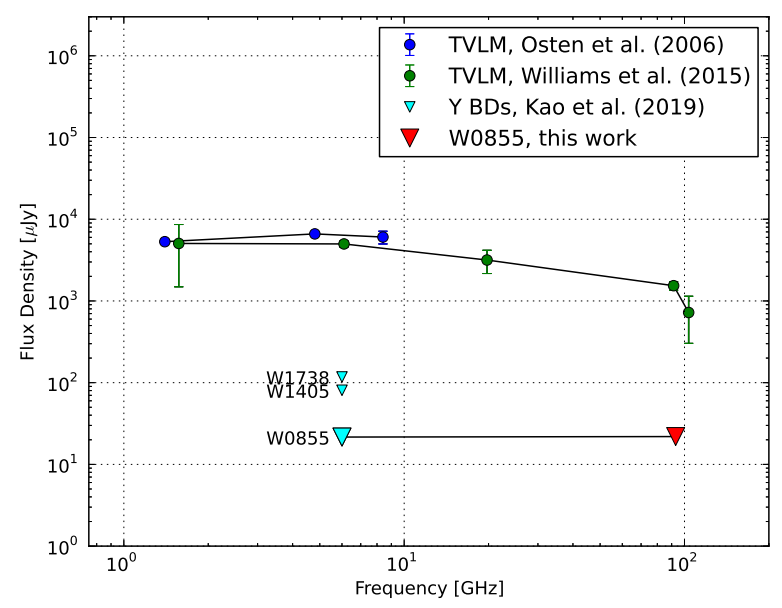

Fig. 3. Radio SED (steady, quiescent emission, Stokes I) of W0855, TVLM 513-46546 (TVLM), and other ultracool dwarfs scaled to the distance of W0855 $(D=2.23 \mathrm{pc})$. See Sect. 3.2 for details.

on its periodic ECMI flares at $4-8 \mathrm{GHz}$ (Hallinan et al. 2006). ECMI, however, was ruled out as the origin of the $\mathrm{mm}$ emission detected later (Williams et al. 2015). Instead, Williams and collaborators argued that the non-flaring emission at $95 \mathrm{GHz}$ stems from gyrosynchrotron processes in an ambient magnetic field $\geq 40 \mathrm{G}$.

Other direct methods for measuring the magnetic fields of W0855 are not feasible: we cannot follow Reiners \& Basri (2006, 2007) and Shulyak et al. (2017) to measure the field from line broadening because the object is inaccessible in the optical.

Figure 3 compares the upper limit on the steady emission from W0855 from our work and from Kao et al. (2019) with the radio SED of the M9 dwarf TVLM 513 (Osten et al. 2006; Williams et al. 2015) and upper limits for other Y type BDs (Kao et al. 2019), all scaled to the distance of W0855. Clearly, W0855 has the most stringent limit for an object of this type.

\section{Summary and conclusions}

We report deep (94 min exposure) ALMA $3 \mathrm{~mm}$ observations of W0855, an ultracool brown dwarf at $\approx 2$ pc. The object was neither detected as a steady nor as a pulsed emitter. We derive a $3 \sigma$ upper limit of $21.9 \mu \mathrm{Jy}$ on the steady flux at $85.1-100.9 \mathrm{GHz}$. Likewise, a search for pulsed emission in bins of $30 \mathrm{~s}$ time resolution across our two nearly consecutive $47 \mathrm{~min}$ on-source observation time windows did not result in a detection. We place a $3 \sigma$ upper limit on the pulsed Stokes $I$ emission at $264 \mu \mathrm{Jy}$. Even though both our upper limits are nominally at the same flux level as the corresponding limits derived from the recent non-detection of steady and pulsed emission at $4-8 \mathrm{GHz}$ by Kao et al. (2019), they are less constraining because the power-law spectrum measured in similar objects such as TVLM 513 suggests that an index $<0$ (see Fig. 3) between $8 \mathrm{GHz}$ and $85 \mathrm{GHz}$ should be assumed for W0855.

W0855 is obviously not a prime candidate for the study of magnetospherically active ultracold BDs. Still, because it is so close to Earth, it may be justified to carry out an extremely deep observing campaign with sensitivities at the $0.5 \mu \mathrm{Jy}$ level in order to characterise our unique neighbour. This should first be done in the 4-8 GHz regime or possibly with the new ALMA Band $1(35-50 \mathrm{GHz})$, which is presently under construction. In addition, longer-term monitoring of this and other ultracool dwarfs at moderate sensitivity is needed to explore variability on timescales longer than a few hours.
Acknowledgements. This paper makes use of the following ALMA data: ADS/JAO.ALMA\#2019.1.00202.S. ALMA is a partnership of ESO (representing its member states), NSF (USA) and NINS (Japan), together with NRC (Canada), MOST and ASIAA (Taiwan), and KASI (Republic of Korea), in cooperation with the Republic of Chile. The Joint ALMA Observatory is operated by ESO, AUI/NRAO and NAOJ.

\section{References}

ALMA Pipeline Team 2019, ALMA Science Pipeline User's Guide, ALMA Doc, 7, 13

Anglada-Escudé, G., Amado, P. J., Barnes, J., et al. 2016, Nature, 536, 437 Beamín, J. C., Ivanov, V. D., Bayo, A., et al. 2014, A\&A, 570, L8 Berger, E. 2002, ApJ, 572, 503

Berger, E., Ball, S., Becker, K. M., et al. 2001, Nature, 410, 338 Beichman, C., Gelino, C. R., Kirkpatrick, J. D., et al. 2013, ApJ, 764, 101 Bower, G. C., Bolatto, A., Ford, E. B., \& Kalas, P. 2009, ApJ, 701, 1922 Burningham, B., Hardcastle, M., Nichols, J. D., et al. 2016, MNRAS, 463, 2202

Chauvin, G., Lagrange, A.-M., Dumas, C., et al. 2004, A\&A, 425, L29 Chauvin, G., Lagrange, A.-M., Dumas, C., et al. 2005, A\&A, 438, L25 Curiel, S., Ortiz-León, G. N., Mioduszewski, A. J., et al. 2019, ApJ, 884, 13 Douglas, J. N., Bash, F. N., Bozyan, F. A., et al. 1996, AJ, 111, 1945 Emonts, B., Raba, R., \& Moellenbrock, G. 2019, Proc. ADASS XXIX, ASP Conf. Ser., submitted, [arXiv: 1912.09437]

Esplin, T. L., \& Luhman, K. L. 2016, AJ, 151, 9

Faherty, J. K., Tinney, C. G., Skemer, A., et al. 2014, ApJ, 793, L16

Faherty, J. K., Gagné, J., Burgasser, A. J., et al. 2018, ApJ, 868, 44

Forbrich, J., \& Berger, E. 2009, ApJ, 706, L205

Forbrich, J., Berger, E., \& Reid, M. J. 2013, ApJ, 777, 70

Gawroński, M. P., Goździewski, K., \& Katarzyński, K. 2017, MNRAS, 466, 4211

Gawroński, M. P., Goździewski, K., Katarzyński, K., et al. 2018, MNRAS, 475, 1399

Grodent, D., Clarke, J. T., Kim, J., et al. 2003, J. Geophys. Res. (Space Phys.), 108,1389

Guedel, M., \& Benz, A. O. 1993, ApJ, 405, L63

Hallinan, G., Antonova, A., Doyle, J. G., et al. 2006, ApJ, 653, 690

Hallinan, G., Bourke, S., Lane, C., et al. 2007, ApJ, 662, L25

Hallinan, G., Antonova, A., Doyle, J. G., et al. 2008, ApJ, 684, 644 Hill, T. W. 1979, J. Geophys. Res., 84, 6554

Kao, M. M., Hallinan, G., Pineda, J. S., et al. 2016, ApJ, 818, 24

Kao, M. M., Hallinan, G., \& Pineda, J. S. 2019, MNRAS, 487, 1994

Kirkpatrick, J. D., Martin, E. C., Smart, R. L., et al. 2019, ApJS, 240, 19

Kopytova, T. G., Crossfield, I. J. M., Deacon, N. R., et al. 2014, ApJ, 797, 3

Kunde, V., Hanel, R., Maguire, W., et al. 1982, ApJ, 263, 443

Luhman, K. L. 2013, ApJ, 767, L1

Luhman, K. L. 2014, ApJ, 786, L18

Luhman, K. L., \& Esplin, T. L. 2016, AJ, 152, 78

Marocco, F., Caselden, D., Meisner, A. M., et al. 2019, ApJ, 881, 17

Masci, F. 2011, WISE Internal Memorandum "Computing Flux Upper-limits for Non-detections"

Meisner, A. M., Caselden, D., Kirkpatrick, J. D., et al. 2020, ApJ, 889, 74

Melso, N. D., Kaldon, K. M., \& Luhman, K. L. 2015, AJ, 150, 62

Morley, C. V., Skemer, A. J., Allers, K. N., et al. 2018, ApJ, 858, 97

Nichols, J. D., Burleigh, M. R., Casewell, S. L., et al. 2012, ApJ, 760, 59

Osten, R. A., Hawley, S. L., Bastian, T. S., et al. 2006, ApJ, 637, 518

Pavlenko, Y. V., Jones, H. R. A., Martín, E. L., et al. 2007, MNRAS, 380, 1285

Petry, D., Vila-Vilaro, B., \& Villard, E. 2014, Proc. SPIE, 91520J

Reiners, A., \& Basri, G. 2006, ApJ, 644, 497

Reiners, A., \& Basri, G. 2007, ApJ, 656, 1121

Remijan, A., Biggs, A., Cortes, P., et al. 2019, ALMA Cycle 7 Technical Handbook, ALMA Doc 7.3, ver. 1.1

Scholz, R.-D. 2014, A\&A, 561, A113

Sheehan, P. D., Wu, Y.-L., Eisner, J. A., et al. 2019, ApJ, 874, 136

Shulyak, D., Reiners, A., Engeln, A., et al. 2017, Nat. Astron., 1, 0184

Skemer, A. J., Morley, C. V., Allers, K. N., et al. 2016, ApJ, 826, L17

Tinney, C. G., Kirkpatrick, J. D., Faherty, J. K., et al. 2018, ApJS, 236, 28

Treumann, R. A. 2006, A\&ARv, 13, 229

Williams, P. K. G., Casewell, S. L., Stark, C. R., et al. 2015, ApJ, 815, 64

Williams, P. K. G. 2018, Handbook of Exoplanets, 171

Wright, E. L., Eisenhardt, P. R. M., Mainzer, A. K., et al. 2010, AJ, 140, 1868

Wright, E. L., Mainzer, A., Kirkpatrick, J. D., et al. 2014, AJ, 148, 82

Xu, S., Zhang, B., Reid, M. J., et al. 2019, ApJ, 875, 114

Zapatero Osorio, M. R., Lodieu, N., Béjar, V. J. S., et al. 2016, A\&A, 592, A80

Zic, A., Lynch, C., Murphy, T., et al. 2019, MNRAS, 483, 614 